American Medical Journal 3 (2): 75-81, 2012

ISSN 1949-0070

(C) 2012 Science Publications

\title{
Coronary Endothelial Dysfunction after Drug-Eluting Stent Implantation
}

\author{
Shigenori Ito \\ Division of Cardiovascular Medicine, \\ Nagoya City East Medical Center 1-2-23 Wakamizu, \\ Chikusa-Ku, Nagoya-Shi, Aichi-Ken, 464-857, Japan
}

\begin{abstract}
Problem statement: Stent thrombosis, which may occur even one year after stent implantation, is a drawback of Drug-Eluting Stents (DES). Among the variety of causes of stent thrombosis after DES implantation, coronary endothelial dysfunction manifested or exaggerated by DES implantation should not be overlooked. This review article summarizes previous reports on coronary endothelial dysfunction after DES implantation and/or coronary spasm and discusses the mechanism, clinical implications and prognosis. Although novel stents that do not impair endothelial function will most likely be developed in the near future, millions of patients have already undergone implantation of a first or second generation DES. Conclusion/Recommendations: Some studies have reported that a drug improves the impairment of endothelial function. On the other hand, no direct relationships between spasm and stent thrombosis after DES implantation have been reported. A larger study is warranted to clarify the clinical significance of this important clinical issue.
\end{abstract}

Key words: Drug-Eluting Stents (DES), Bare-Metal Stent (BMS), Acetylcholine (ACH)

\section{INTRODUCTION}

Endothelial cells produce many vaso-active substances that maintain vascular homeostasis and normal vasomotor tone. Nitric oxide is a key factor generated by endothelial cells and which mainly determines basal vascular smooth muscle tone and opposes the action of potent endothelium-derived contracting factors such as angiotensin II and endothelin1 (Yanagisawa et al., 1988). Endothelial dysfunction can lead to a variety of pathophysiologic processes, such as vasospasm, vasoconstriction, thrombus formation and abnormal vascular proliferation. In many circumstances, reduced endothelial function has been shown to correlate with increased atherogenesis, as well as cardiovascular risk (Schachinger et al., 2000). Whether DES-induced endothelial dysfunction produces a similar risk is not known (Muhlenstein, 2008).

Causes of stent thrombosis: The mechanisms underlying stent thrombosis are multifactorial and include patient-related factors, procedural factors (including stent choice) and post procedural factors (including type and duration of antiplatelet therapy). From the standpoint of stent technology, potential mechanisms of late stent thrombosis after Drug-Eluting Stent (DES) implantation include delayed or absent endothelialization of the stent struts, hypersensitivity/inflammatory and/or thrombotic reaction to the polymers, strut fractures, late malapposition and the development of neoatherosclerosis within stents with plaque rupture (Kirtane and Stone, 2011; Nakazawa et al., 2011). Delayed endothelialization in the coronary vessels after DES deployment has been closely related to several adverse events, the most severe of which is thrombus formation. Accordingly, this finding could partially explain the increased late thrombotic events observed with the first generation DES.

Stent thrombosis after drug-eluting stent implantation versus bare-metal stent: Stent thrombosis after Bare-Metal Stent (BMS) deployment usually occurs within the first 30 days after deployment and very rarely may occur later. In contrast, stent thrombosis after DES implantation can occur years afterward, with an annual incidence of $0.2-0.6 \%$ in patients with non complex coronary artery disease (Weisz et al., 2009) and 0.4-0.6\% in unrestricted cases (Wenaweser et al., 2008). Thus, stent thrombosis rates increase within the original stent and are higher with DES than BMS, with the differences emerging predominantly beyond the first year after implantation (Stone et al., 2007).

Endothelial dysfunction as a potential cause of stent thrombosis: Coronary vasoconstriction or spasm would result in a reduction of coronary blood flow and deterioration of non-laminar flow within the stented 
vessel, which may be associated with an increase in inflammation and thrombosis (Hamasaki and Tei, 2011).

Endothelial dysfunction after coronary stent implantation: Summary of the reported data on coronary endothelial dysfunction manifested after stent implantation is shown in this chapter. Generally, it is known that DES as well as BMS can provoke and/or or exaggerate endothelial dysfunction after implantation, however, its degree may differ among stent types. In all but 2 reports, an Acetylcholine $(\mathrm{ACH})$ provocation test was adopted to evaluate coronary endothelial dysfunction. I suspect the doses or concentrations of ACh infused may be partially related to the difference in the degree of endothelial dysfunction in the following data.

Bare-metal stent: Spasm may occur around the stent struts even after BMS implantation (Fuke et al., 2007; Tanabe et al., 2002). Enhanced endothelial dysfunction following BMS implantation has been reported (Caramori et al., 1999; Beusekom et al., 1998). The results are slightly different between reports.

\section{Sirolimus-eluting stent (SES, Cypher ${ }^{\mathrm{TM}}$, Cordis} Corp., Miami Lakes, Florida): Recently, concern has been raised that SES may be associated with an increased rate of stent thrombosis owing to delayed or absent endothelialization (Jeremias et al., 2004; Virmani et al., 2004). In this context, Togni et al. (2005) examined coronary endothelial dysfunction by evaluating the coronary vasomotor response to exercise six months after SES implantation using biplane quantitative coronary angiography for the first time in 2005. Maekawa et al. (2006) reported a case of recent anterior myocardial infarction without a history of coronary spasm. A severe vasoconstrictive response was produced by a small dose of intracoronary ACh infusion, indicating severe endothelial dysfunction in the SES at 6 months after stenting. Similar results were reported by Hofma et al. (2006) in a small study using ACh infusion. They evaluated vasoconstriction only in segments distal to the DES and did not clarify if vasoconstriction had existed before SES implantation was not clarified (Hofma et al., 2006). Maekawa et al. (2006) and Fuke et al. (2007) confirmed the feature of a case report (Maekawa et al., 2006) in a study with 21 patients treated with SES and12 patients treated with BMS. In contrast to vasodilation in BMS patients, the response to $\mathrm{ACh}$ in SES patients showed vasoconstriction in the peri-stent area (proximal and distal segments to the implanted stents). They also evaluated the vasoconstrictive response to ACh before SES implantation in a subgroup and found endothelial dysfunction did not exist. It was reported that SES implantation aggravates endothelial vasomotor dysfunction in the infarct-related coronary artery in patients with anteroseptal acute myocardial infarction (Obata et al., 2007). They evaluated in both resistance and epicardial coronary arteries by measuring the diameter and blood flow in response to $\mathrm{ACh}$ infusion (Obata et al., 2007).

Paclitaxel-eluting stent (PES, Taxus ${ }^{\mathrm{TM}}$, Boston Scientific, Natick, Massachusetts): Togni et al. (2007) studied coronary endothelial dysfunction after PES implantation using similar supine bicycle exercise methods in 15 patients with PES deployment comparing 12 patients with BMS as a control group as in a study of SES cases (Togni et al., 2005). PES was associated with exercise-induced vasoconstriction in the persistent (proximal and distal) region suggesting endothelial dysfunction $(-13 \pm 6 \%$ and $-18 \pm 4 \%$, respectively; $\mathrm{p}<0.005 \mathrm{Vs}$ corresponding segments of controls). The degree of vasoconstriction was quite similar to those of SES patients $(-12 \pm 4$ and $-15 \pm 6 \%$; $\mathrm{p}<0.001$, Vs. Corresponding segments of controls) (Togni et al., 2005). In this study, the follow-up period varied between 2-12 months after PES implantation. They observed a time-dependent improvement in vascular function in the pre-stent lesion. Shin et al. (2007) also showed that SES or PES implantation could be associated with the similar pattern of endothelial dysfunction identified predominantly in the long distal portion of the treated vessels compared with BMS. The degree of vasoconstriction was around $25 \%$ (vessel diameter change in comparison of the baseline to after intracoronary ACh infusion) in both distal and far distal segments for both SES and PES groups (Shin et al., 2007). This report is different from previous ones in that far distal segment $(10-20 \mathrm{~mm}$ distal to the stent distal edge) were also evaluated and a similar degree of endothelial dysfunction was observed. Kim et al. (2008) reported similar results in a slightly larger study comparing coronary vasoconstriction of SES and PES with that of BMS. The percent change in vessel diameter at segments $5 \mathrm{~mm}$ distal to the stent in The Left Anterior Descending coronary artery (LAD) was slightly higher than those of previous reports $(-72.9 \pm 11.6 \%$ in SES, $71.8 \pm 15.7$ in PES Vs $-7.91 \pm 20.4$ in BMS) (Kim et al., 2008) by ACh infusion. The endothelial dysfunction in the distal segments appears to be more significant than in the proximal segments after DES implantation. Furthermore, the findings of Shin et al. (2007) and Ito et al. (2001) suggested that the long distal portion could show endothelial dysfunction. 
Biolimus A9-eluting stents (BES, Nobori ${ }^{\mathrm{TM}}$, Terumo, Tokyo, Japan): Delayed healing in association with an increased risk of late and very late stent thrombosis and paradoxical coronary vasoconstriction at coronary segments adjacent to the stent may be attributable to delayed endothelial healing and/or endothelial dysfunction caused by eluting-drug, polymer-induced inflammation, or hypersensitivity reaction. New DES have been developed with different stent designs, drugs and polymers. BES is a second-generation DES that carries a bioresorbable polymer (polylactic acid) from which A9, an analogue of sirolimus, is eluted. Hamilos et al. (2008a) studied 19 patients with a BES and 15 patients with an SES at 9 months after stent implantation. Endothelium dependent and independent coronary vasomotion were tested proximally and distally to the stent as well as at a reference segment during atrial pacing at increasing heart rates. Of the patients with BES, only 2 showed vasoconstriction while 17 showed vasodilation. In contrast, of the patients with an SES, 9 showed vasoconstriction while 6 showed vasodilation. This result may be explained by the different drug release kinetics, design, or characteristics of polymer used in the stent system (Hamilos et al., 2008a). BES seem to improve coronary endothelial dysfunction compared with first generation DES.

Zotarolimus-eluting stents (ZES, Endeavor ${ }^{\mathrm{TM}}$, Medtronic Vascular, Inc., Santa Rosa, California): A prospective, randomized, 6-month comparison of the coronary vasomotor response associated with a ZES versus SES was performed by Kim et al. (2009) to investigate the potential superiority of ZES in terms of uniform and rapid healing of the endothelium in 50 patients. Vasoconstriction in response to $\mathrm{ACh}$ in the peristent region was less pronounced in the ZES group than in the SES group at 6-month follow-up (\% diameter change from baseline: $-71.1 \pm 15.6$ in SES Vs$38.5 \pm 24.4$ in ZES, $-7.99 \pm 6.42$ in BMS at a maximal dose of ACh in the distal segment), which suggests that endothelial function associated with ZES can be more preserved than with SES (Kim et al., 2009). The majority (approximately 95\%) of zotarorimus is released from the stent by 14 days (Kandzari and Leon, 2006). This rapid evolution kinetics of ZES may reduce the local toxicity to the endothelium leading to the possibility of low risk of late stent thrombosis (Gershlick et al., 2007).

Comparison of first generation and second generation DES: Hamilos et al. (2008b) evaluated the influence of BMS and four types of DES on endothelium-dependent vasodilation. Their study revealed that SES and PES showed vasoconstriction, while on the other hand, ZES and BES had vasodilatory responses that were similar to BMS (Hamilos et al., 2008b).

Second generation bioresorbable everolimus-eluting vascular scaffold (ABSORB BVS (Rev.1.1. Abbott Vascular, Santa Clara, California): Potential and/or ongoing stent modification to reduce stent thrombosis have included improving the biocompatibility of the stent and polymer, using bioabsorbable polymers and/or using stent surface modifications to stimulate vascular endothelialization. Bioresorbable vascular scaffold (Dudek et al., 2011; Serruys et al., 2011) will play a major role in a certain subset of coronary lesions in the near future, because bare-metal stents, which contain no eluting drug or polymer have been reported to cause chronic inflammation several years after implantation (Inoue et al., 2004), resulting in very late ISR or stent thrombosis. In a recent study by Serruys et al. (2011) pharmacological vasomotion by methylergonovine $(\mathrm{N}$ $=13$ ) showed some degree of vasoconstriction in 12 patients. ACH $(\mathrm{N}=24)$ showed vasodilation of the scaffolded segment in 8, 1 had unchanged lumen dimension and 10 patients showed vasoconstriction of the scaffolding segments (5 excluded) (Serruys et al., 2011). These findings suggest that full coverage by endothelial cells and complete return of functional capacity of these cells is not yet achieved in the majority of the patients who underwent second generation ABSORB BVS at 12 months. Vasomotion was assessed by measuring changes in mean lumen diameter in the scaffolding segments and in the $5-\mathrm{mm}$ proximal and 5-mm distal adjacent segments.

Mechanism of coronary endothelial dysfunction at distal segments: Approximately $80 \%$ of the sirolimus is released within 30 days of implantation.

It is, therefore, unlikely that the drug can affect vasomotion in the distal segment by diffusion from the bloodstream later after implantation. However, we cannot exclude the possibility that the drug may reach the vessel wall directly distal to a drug-eluting stent, for example by diffusion through the tissue and through the vasa vasorum (Hofma et al., 2006) thus, recovery of endothelial function in the distal portion may be delayed due to the effects of these drugs (Shin et al., 2007). Inoue et al. (2007) revealed that CD34-positive bone marrow-derived stem cells were mobilized after BMS implantation but strongly suppressed after SES implantation in the human stent model. The inhibition of these cellular responses by DES may lead to the coronary endothelial dysfunction. Coronary 
vasoconstricting responses to serotonin (10 and 100 $\mu \mathrm{g} / \mathrm{kg}$ intracoronary administration) were significantly enhanced at the PES site compared with the BMS site in a porcine model $(45 \pm 4 \%$ Vs. $30 \pm 3 \%, \mathrm{p}<0.01 ; \mathrm{n}=12$ each) and were abolished by hydroxyfasudil (90 and $300 \mu \mathrm{g} / \mathrm{kg}$ intracoronary administration), a selective Rho-kinase inhibitor (Shiroto et al., 2009). This study was performed in normal juvenile pigs without preexisting atherosclerotic coronary lesions. This might explain, at least in part, the discrepancy between this animal study (normal vascular function of the distal segment) and another previous clinical study that showed coronary hyperconstricting responses even at the distal segments of DES implanted arteries (Shiroto et al., 2009). They also revealed that paclitaxel significantly enhanced Rho-kinase expression and activity in human coronary artery smooth muscle cells as a baseline molecular mechanism (Shiroto et al., 2009). Most of the previous studies did not mention coronary vasospasms, which can be the strongest manifestation of coronary endothelial dysfunction of the entire coronary bed. On the other hand, coronary vasospastic angina has ethnic characteristics and has higher frequencies in Asian countries than in Caucasian countries (Pristipino et al., 2000; Beltrame et al., 1999). In fact, in a couple of reports from Asian countries, the coronary endothelial dysfunction after DES implantation may have been more severe than that in other studies from European countries and the United States (Fuke et al., 2007; Maekawa et al., 2006; Ito et $a l ., 2011)$. Thus, an intrinsic vasospastic property might be exaggerated by the effect of DES implantation or DES-induced hyperconstriction that predominantly occurs in individuals who are a priori predisposed to abnormal vasomotion after DES implantation even if the patients with obvious positive results in an ACh provocation test were excluded before entry. There might be a relationship between delayed or absent healing (endothelialization) of stent struts and endothelial dysfunction in the distal segment to the stents. Fujii et al. (2011) showed the correlation between percentage of covered stent struts and endothelial dysfunction in patients who underwent ZES implantation. However, we should wait to draw any definite conclusions because the mechanism of coronary dysfunction has not been clarified.

Angiographic and clinical features of coronary endothelial dysfunction after DES implantation in real world patients: Most studies evaluated coronary vasomotion in the peri-stent coronary segment for one lesion with one drug-eluting stent. The angiographic and clinical characteristics of real-world patients with coronary spasm following DES implantation have not been well documented. We performed a study that included all patients at our hospital who underwent coronary angiography at follow-up after DES deployment (Cypher and Taxus stents) between July 2007 and March 2009 (Ito et al., 2011). We performed an ACh provocation test for diagnosing coronary vasospasm in three vessels, excluding patients with significant stenosis. ACh provocation test was positive in $65.4 \%(36 / 55)$ of the coronary arteries and in $71.4 \%$ (30/42) of the patients with drug-eluting stents. There was no difference in the positive rate between patients with and without symptoms. A total of $65.0 \%$ (13/20) of asymptomatic patients also showed positive results. In patients with positive results in the ACh provocation test, vasoconstriction at segments distal to the stent was exaggerated compared with corresponding segments in non-stented vessels $(0.46 \pm 0.27$ Vs $0.31 \pm 0.20, p=$ 0.008). Vessels with positive results had a long stent length compared with those with negative results $(31.6 \pm 13.6 \mathrm{~mm}$ Vs $24.2 \pm 11.2 \mathrm{~mm}, \mathrm{p}=0.049)$. We concluded that the coronary vasoconstriction was exaggerated at distal segments in DES-implanted vessels compared to non-stented vessel segments and stent length was longer in the ACh provocation test positive group. In a few reports from Asian countries, the arterial response to ACh appeared to be strong, indicating the possibility of coronary vasospasm. Thus, we examined the frequency of provoking spasm in the entire coronary bed as well as Peoria-stent vasoconstriction by QCA. The peri-stent vasoconstriction was stronger in the distal segment than in the proximal segment. No case had spasm localized in the peri-stent segment. In almost all cases, strong peri-stent vasoconstriction was associated with spasm in the far distal bed. Obata et al. (2007) reported on the incidence of stent-edge spasm after bare-metal stent implantation. The spasm was provoked in $19.2 \%$ of the patients with vasospastic angina $6.4 \pm 2.0$ months after stent implantation. If moderate stent-edge spasm was included, stent-edge spasm occurred in $38.4 \%$ of these patients. Even in patients without vasospastic angina, the rate was $30.4 \%$. In our study, the pattern of spasm after DES implantation was different to that in bare-metal stents. This might be due to the pathogenesis of ACh induced spasm after DES implantation.

Strategies to improve coronary dysfunction in patients who successfully underwent DES implantation: In the study by Kitahara et al. (2011) the beneficial effect of pioglitazone on coronary dysfunction was observed in the segment distal to the SES but not to the segment proximal to the SES. Sirolimus from the SES moves with blood flow. Thus, 
endothelial dysfunction might be more pronounced in segments distal to the SES than proximity to the SES (Kitahara et al., 2011). Calcium channel blockers may be useful in patients with coronary endothelial dysfunction detected at follow-up coronary angiography as in coronary spastic angina. It has been reported that 4 major calcium channel blockers generally suppress VSA attacks and benidipine was found to have a more pronounced inhibitory effect on cardiovascular events in a meta-analysis with a total of 1,997 vasospastic angina patients (Nishigaki et al., 2010).

Inflammation and vasospastic angina: In patients with vasospastic angina, plasma level of hsCRP, a sensitive marker of inflammation, increase compared with those without spasm (Hung et al., 2005; Itoh et al., 2007). A recent study demonstrated that 6-month treatment with a statin (fluvastatin) significantly reduced the occurrence of coronary spasm along with the decrease in hs CRP levels (Yasue et al., 2008). As a marker of coronary spasm, Rho-kinase activity in circulating leucocytes was significantly increased in the vasospastic angina group, suggesting that the Rho-kinase activity could be a more sensitive serological marker of coronary spasm than hs CRP (Kikuchi et al., 2011). These markers might have potential clinical usefulness even for endothelial dysfunction after DES implantation.

Prognosis of coronary endothelial dysfunction after DES implantation: It should be elucidated whether coronary endothelial dysfunction has prognostic significance in clinical cardiac practice. There is a paucity of data concerning this important topic. To the best of my knowledge, no reports have shown a direct relationship between coronary endothelial dysfunction or spasm and late stent thrombosis. Although absent or delayed re-endothelialization is the most important cause of stent thrombosis, impairment of endothelial cells within a DES might also cause stent thrombosis. We (Ito et al., 2011) reported a mid-term follow-up study in a small group with 55 patients who underwent ACh provocation test at follow-up coronary angiography after first generation DES implantation. The mean follow-up period was $23.9 \pm 4.5$ months. There was no definite stent thrombosis. For patients who complained of angina were treated medically. One patient died of unknown cause 7 months after the ACh provocation test. This patient underwent PCI for advanced stenosis in the right coronary artery 1 month after the test. One patient suffered a cerebral infarction after discontinuation of antiplatelet therapy and another patient experienced cerebral bleeding after falling down. All 7 patients described above had positive results in $\mathrm{ACh}$ provocation test. The frequency of angina was not different between patients with positive and negative results in the ACh provocation test (4/30 Vs $0 / 12, p=0.308)$. The incidence of cardiac events was relatively low and not different between patients with positive and negative results in ACh provocation test. The symptoms of angina observed in 4 patients might have been related to the coronary spasm that occurred after DES implantation. Thus, a larger study is warranted to clarify the clinical significance of this important clinical issue.

\section{REFERENCES}

Beltrame, J.F., S. Sasayama and A. Maseri, 1999. Racial heterogeneity in coronary artery vasomotor reactivity: Differences between Japanese and Caucasian patients. J. Am. Coll. Cardiol., 33: 14421452. DOI: 10.1016/S0735-1097 (99)00073-X

Beusekom, H.M.V., D.M. Whelan, S.H. Hofma, S.C. Krabbendam and V.W.V. Hinsbergh et al., 1998. Long-term endothelial dysfunction is more pronounced after stenting than after balloon angioplasty in porcine coronary arteries. J. Am. Coll. Cardiol., 32: 1109-1117. DOI: 10.1016/S0735-1097 (98)00348-9

Caramori, P.R.A., V.C. Lima, P.H. Seidelin, G.E. Newton and J.D. Parker et al., 1999. Long-term endothelial dysfunction after coronary artery stenting. J. Am. Coll. Cardiol., 34: 1675-1679. DOI: 10.1016/S0735-1097 (99)00411-8

Dudek, D., Y. Onuma, J.A. Ormiston, L. Thuesen and K. Miquel-Hebert et al., 2011. Four-year clinical follow-up of the ABSORB everolimus-eluting bioresorbable vascular scaffold in patients with de novo coronary artery disease: the ABSORB trial. EuroIntervention, 7: 1060-1061. PMID: 21959320

Fujii, K., D. Kawasaki, K. Oka, H. Akahori and M. Fukunaga et al., 2011. Endothelium-dependent coronary vasomotor response and neointimal coverage of zotarolimus-eluting stents 3 months after implantation. Heart, 97: 977-982. DOI: 10.1136/hrt.2010.204594

Fuke, S., K. Maekawa, K. Kawamoto, H. Saito and T. Sato et al., 2007. Impaired endothelial vasomotor function after sirolimus-eluting stent implantation. Circe. J., 71: 220-225. PMID: 17251671

Gershlick, A., D.E. Kandzari, M.B. Leon, W. Wijns and I.T. Meredith et al., 2007. Zotarolimus-eluting stents in patients with native coronary artery disease: Clinical and angiographic outcomes in 1,317 patients. Am. J. Cardiol., 100: 45M-55M. DOI: 10.1016/j. amjcard. 2007.08.021 
Hamasaki, S. and C. Tei, 2011. Effect of coronary endothelial functions on outcomes in patients undergoing percutaneous coronary intervention. J. Cardiol., 57: 231-238. DOI: 10.1016/j.jjcc.2011.02.003

Hamilos, M.I., M. Ostojic, B. Beleslin, D. Sagic and L. Mangovski et al., 2008a. Differential effects of drug-eluting stents on local endothelium-dependent coronary vasomotion. J. Am. Coll. Cardiol., 51: 2123-2129. DOI: 10.1016/j.jacc.2007.12.059

Hamilos, M., J. Sarma, M. Ostojic, T. Cuisset and G. Sarno et al., 2008b. Interference of drug-eluting stents with endothelium-dependent coronary vasomotion: Evidence for device-specific responses. Circ. Cardiovasc Interv., 1: 193-200. DOI:

10.1161/CIRCINTERVENTIONS.108.797928

Hofma, S.H., W.J.V.D. Giessen, B.M.V. Dalen, P.A. Lemos and E.P. McFadden et al., 2006. Indication of long-term endothelial dysfunction after sirolimus-eluting stent implantation. Eur. Heart J., 27: 166-170. DOI: 10.1093/eurheartj/ehi571

Hung, M.J., W.J. Cherng, N.I. Yang, C.W. Cheng and L.F. Li, 2005. Relation of high-sensitivity Creactive protein level with coronary vasospastic angina pectoris in patients without hemodynamically significant coronary artery disease. Am. J. Cardiol., 96: 1484-1490. DOI: 10.1016/j. amjcard. 2005.07.055

Inoue, K., K. Abe, K. Ando, S. Shirai and K. Nishiyama et al., 2004. Pathological analyses of long-term intracoronary Palmaz-Schatz stenting: Is its efficacy permanent? Cardiovascular Pathol., 13: 109-115. DOI: 10.1016/S1054-8807 (03)00132-7

Inoue, T., M. Sata, Y. Hikichi, R. Sohma and D. Fukuda et al., 2007. Mobilization of CD34-positive bone marrow-derived cells after coronary stent implantation: Impact on restenosis. Circulation, 115: 553-561. DOI: 10.1161/CIRCULATIONAHA.106.621714

Ito, S., K. Nakasuka, K. Morimoto, M. Inomota and T. Yoshida et al., 2001. Angiographic and clinical characteristics of patients with acetylcholineinduced coronary vasospasm on follow-up coronary angiography following drug-eluting stent implantation. J. Invasive Cardiol., 23: 57-64. PMID: 21297201

Ito, S., K. Nakasuka, K. Morimoto, M. Inomata and T. Yoshida et al., 2011. Angiographic and clinical characteristics of patients with acetylcholineinduced coronary vasospasm on follow-up coronary angiography following drug-eluting stent implantation. J. Invasive Cardiol., 23: 57-64. PMID: 21297201
Itoh, T., Y. Mizuno, E. Harada, M. Yoshimura and H. Ogawa et al., 2007. Coronary spasm is associated with chronic low-grade inflammation. Circ. J., 71: 1074-1078. PMID: 17587713

Jeremias, A., B. Sylvia, J. Bridges, A.J. Kirtane and B. Bigelow et al., 2004. Stent thrombosis after successful sirolimus-eluting stent implantation. Circulation, 109: 1930-1932. DOI: 10.1161/01.CIR.0000127105.99982.21

Kandzari, D.E. and M.B. Leon, 2006. Overview of pharmacology and clinical trials program with the zotarolimus-eluting endeavor stent. J. Interv. Cardiol., 19: 405-413. DOI: 10.1111/j. 15408183.2006.00184. $\mathrm{x}$

Kikuchi, Y., S. Yasuda, K. Aizawa, R. Tsuburaya and Y. Ito et al., 2011. Enhanced Rho-kinase activity in circulating neutrophils of patients with vasospastic angina: A possible biomarker for diagnosis and disease activity assessment. J. Am. Coll. Cardiol., 58: 1231-1237. DOI: 10.1016/j.jacc.2011.05.046

Kim, J.W., S.Y. Suh, C.U. Choi, J.O. Na and E.J. Kim et al., 2008. Six-month comparison of coronary endothelial dysfunction associated with sirolimuseluting stent versus Paclitaxel-eluting stent. JACC Cardiovasc Interv., 1: 65-71. DOI: 10.1016/j.jcin.2007.11.002

Kim, J.W., H.S. Seo, J.H. Park, J.O. Na and C.U. Choi et al., 2009. A prospective, randomized, 6-month comparison of the coronary vasomotor response associated with a zotarolimus-versus a sirolimuseluting stent: Differential recovery of coronary endothelial dysfunction. J. Am. Coll. Cardiol., 53: 1653-1659. DOI: 10.1016/j.jacc.2009.01.051

Kirtane, A.J. and G.W. Stone, 2011. How to minimize stent thrombosis. Circulation, 124: 1283-1287. DOI: 10.1161/CIRCULATIONAHA.110.976829

Kitahara, H., Y. Kobayashi, Y. Iwata, Y. Fujimoto and I. Komuro, 2011. Effect of pioglitazone on endothelial dysfunction after sirolimus-eluting stent implantation. Am. J. Cardiol., 108: 214-219. DOI: 10.1016/j.amjcard.2011.03.029

Maekawa, K., K. Kawamoto, S. Fuke, R. Yoshioka and H. Saito et al., 2006. Severe endothelial dysfunction after sirolimus-eluting stent implantation. Circulation, 113: e850-e-851. DOI: 10.1161/CIRCULATIONAHA. 105.597948

Muhlenstein, J.B., 2008. Endothelial dysfunction associated with drug-eluting stents what, where, when and how? J. Am. Coll. Cardiol., 51: 21392140. PMID: 18510960

Nakazawa, G., F. Otsuka, M. Nakano, M. Vorpahl and S.K. Yazdani et al., 2011. The pathology of neoatherosclerosis in human coronary implants: Bare-metal and drug-eluting stents. J. Am. Coll. Cardiol., 57: 1314-1322. DOI: 10.1016/j.jacc.2011.01.011 
Nishigaki, K., Y. Inoue, Y. Yamanouchi, Y. Fukumoto and S. Yasuda et al., 2010. Prognostic effects of calcium channel blockers in patients with vasospastic angina--a meta-analysis. Circ. J., 74: 1943-1950. PMID: 20668353

Obata, J., Y. Kitta, H. Takano, Y. Kodama and T. Nakamura et al., 2007. Sirolimus-eluting stent implantation aggravates endothelial vasomotor dysfunction in the infarct-related coronary artery in patients with acute myocardial infarction. J. Am. Coll. Cardiol., 50: 1305-1309. DOI: 10.1016/j.jacc.2007.06.031

Pristipino, C., J.F. Beltrame, M.L. Finocchiaro, R. Hattori and M. Fujita et al., 2000. Major racial differences in coronary constrictor response between Japanese and Caucasians with recent myocardial infarction. Circulation, 101: 1102-1108. PMID: 10715255

Schachinger, V., M.B. Britten and A. Zeiher, 2000. Impaired epicardial coronary vasoreactivity predicts for adverse cardiovascular events during long-term follow up. Circulation, 101: 1899-1906.

Serruys, P.W., Y. Onuma, D. Dudek, P.C. Smits and J. Koolen et al., 2011. Evaluation of the second generation of a bioresorbable everolimus-eluting vascular scaffold for the treatment of de novo coronary artery stenosis: 12-month clinical and imaging outcomes. J. Am. Coll. Cardiol., 58: 15781588. DOI: $10.1016 /$ j.jacc.2011.05.050

Shin, D.I., P.J. Kim, K.B. Seung, D.B. Kim and M.J. Kim et al., 2007. Drug-eluting stent implantation could be associated with long-term coronary endothelial dysfunction. Int. Heart J., 48: 553-567. PMID: 17998765

Shiroto, T., S. Yasuda, R. Tsuburaya, Y. Ito and J. Takahashi et al., 2009. Role of Rho-Kinase in the pathogenesis of coronary hyperconstricting responses induced by drug-eluting stents in pigs in vivo. J. Am. Coll. Cardiol., 54: 2321-2329. DOI: 10.1016/j.jacc.2009.07.045

Stone, G.W., J.W. Moses, S.G. Ellis, J. Schofer and K.D. Dawkins et al., 2007. Safety and efficacy of sirolimus- and paclitaxel-eluting coronary stents. N Engl. J. Med., 356: 998-1008. DOI: 10.1056/NEJMoa067193
Tanabe, Y., E. Itoh, K. Suzuki, M. Ito and Y. Hosaka et $a l ., 2002$. Limited role of coronary angioplasty and stentingin coronary spastic angina with organic stenosis. J. Am. Coll. Cardiol., 39: 1120-1126. DOI: 10.1016/S0735-1097 (02)01746-1

Togni, M., S. Windecker, R. Cocchia, P. Wenaweser and S. Cook et al., 2005. Sirolimus-eluting stents associated with paradoxical coronary vasoconstriction. J. Am. Coll. Cardiol., 46: 231236. DOI: $10.1016 /$ j.jacc.2005.01.062

Togni, M., L. Raber, R. Cocchia, P. Wenaweser and S. Cook et al., 2007. Local vascular dysfunction after coronary paclitaxel-eluting stent implantation. Int. J. Cardiol., 120: 212-220. DOI: 10.1016/j. ijcard. 2006.09.021

Virmani, R., G. Guagliumi, A. Farb, G. Musumeci and N. Grieco et al., 2004. Localized hypersensitivity and late coronary thrombosis secondary to a sirolimus-eluting stent: Should we be cautious? Circulation, 109: 701-705. DOI: 10.1161/01.CIR.0000116202.41966.D4

Weisz, G., M.B. Leon, D.R.J. Holmes, D.J. Kereiakes and J.J. Popma et al., 2009. Five-year follow-up after sirolimus-eluting stent implantation: Results of the SIRIUS (sirolimus-eluting stent in de-Novo native coronary lesions) trial. J. Am. Coll. Cardiol., 53: 1488-1497. DOI: 10.1016/j.jacc.2009.01.050

Wenaweser, P., J. Daemen, M. Zwahlen, R. Van Domburg and P. Juni et al., 2008. Incidence and correlates of drug-eluting stent thrombosis in routine clinical practice: 4-year results from a large 2-institutional cohort study. J. Am. Coll Cardiol., 2008: 1134-1140. DOI: 10.1016/j.jacc.2008.07.006

Yanagisawa, M., H. Kurihara, S. Kimura, Y. Tomobe and M. Kobayashi et al., 1988. A novel potent vasoconstrictor peptide produced by vascular endothelial cells. Nature, 332: 411-415. DOI: $10.1038 / 332411 \mathrm{a} 0$

Yasue, H., Y. Mizuno, E. Harada, T. Itoh and $\mathrm{H}$. Nakagawa et al., 2008. Effects of a 3-hydroxy-3methylglutaryl coenzyme A reductase inhibitor, fluvastatin, on coronary spasm after withdrawal of calcium-channel blockers. J. Am. Coll Cardiol., 51: 1742-1748. DOI: 10.1016/j.jacc.2007.12.049 